\title{
Brain Diffusion Abnormalities in Children with Tension-Type and Migraine-Type Headaches
}

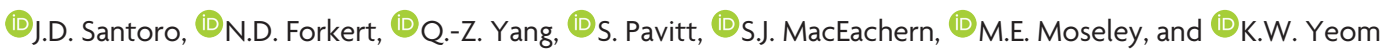

\begin{abstract}
BACKGROUND AND PURPOSE: Tension-type and migraine-type headaches are the most common chronic paroxysmal disorders of childhood. The goal of this study was to compare regional cerebral volumes and diffusion in tension-type and migraine-type headaches against published controls.
\end{abstract}

MATERIALS AND METHODS: Patients evaluated for tension-type or migraine-type headache without aura from May 2014 to July 2016 in a single center were retrospectively reviewed. Thirty-two patients with tension-type headache and 23 with migraine-type headache at an average of 4 months after diagnosis were enrolled. All patients underwent DWI at 3T before the start of pharmacotherapy. Using atlas-based DWI analysis, we determined regional volumetric and diffusion properties in the cerebral cortex, thalamus, caudate, putamen, globus pallidus, hippocampus, amygdala, nucleus accumbens, brain stem, and cerebral white matter. Multivariate analysis of covariance was used to test for differences between controls and patients with tension-type and migraine-type headaches.

RESULTS: There were no significant differences in regional brain volumes between the groups. Patients with tension-type and migrainetype headaches showed significantly increased ADC in the hippocampus and brain stem compared with controls. Additionally, only patients with migraine-type headache showed significantly increased ADC in the thalamus and a trend toward increased ADC in the amygdala compared with controls.

CONCLUSIONS: This study identifies early cerebral diffusion changes in patients with tension-type and migraine-type headaches compared with controls. The hypothesized mechanisms of nociception in migraine-type and tension-type headaches may explain the findings as a precursor to structural changes seen in adult patients with chronic headache.

ABBREVIATIONS: ICHD-3 = International Classification of Headache Disorders, Version 3.0; $\mathrm{MTH}=$ migraine-type headache; $\mathrm{TTH}=$ tension-type headache

M igraine-type headache (MTH) is a common and chronic condition with multifactorial neurovascular etiologies characterized by recurrent paroxysmal attacks of throbbing headache with or without autonomic nervous system dysfunction. ${ }^{1}$ Along with tension-type headache (TTH), these are the most common paroxysmal disorders of childhood. Neuroimaging in

Received October 27, 2017; accepted after revision January 1, 2018.

From the Department of Neurology (J.D.S., Q.-Z.Y., S.P.), Division of Child Neurology, and Department of Radiology (M.E.M.), Stanford University School of Medicine, Stanford, California; Departments of Radiology (N.D.F.) and Pediatrics (S.J.M.) Cumming School of Medicine, University of Calgary, Alberta, Canada; and Department of Radiology (K.W.Y.), Lucile Packard Children's Hospital, Stanford University, Stanford, California.

Paper previously presented in part at: Annual Meeting of the Child Neurology Society, October 4-7, 2017; Kansas City, Missouri.

Please address correspondence to Kristen Yeom, MD, Department of Radiology, Lucile Packard Children's Hospital at Stanford, Stanford University School of Medicine, 725 Welch Rd, MC 5654, Palo Alto, CA 94304; e-mail: kyeom@stanford.edu

http://dx.doi.org/10.3174/ajnr.A5582 these conditions is frequently low yield for clinically relevant findings, with most studies not affecting clinical management. ${ }^{2,3}$

Neuroimaging in adult patients with MTH with aura has previously demonstrated evidence of subcortical lesions in the deep white matter on DWI. ${ }^{4-7}$ These studies have been replicated in pediatric populations with similar results and have also identified volumetric white matter changes throughout the brain. ${ }^{8}$ The typical fingerprint of degenerated neuronal tissue is an increase of ADC. However, previous studies investigating diffusion properties in the brain have yielded variable, often transient, findings in adults. ${ }^{5,9,10}$ Given the chronic activation of nociceptive circuits in TTH and MTH and the possible vascular component of $\mathrm{MTH}$, whether these entities are associated with global or regional ADC alterations in the brain tissue is unknown.

In this study, we conducted a retrospective, cross-sectional analysis of changes in the ADC and regional brain volume in various cerebral regions in healthy pediatric controls and in patients with TTH and MTH younger than 18 years of age with prior 
anatomically normal neuroimaging examination findings. We hypothesized that pediatric patients with MTH would differ from healthy controls and patients with TTH in regional ADC properties in limbic and brain stem structures due to the presumed differences in the pathophysiology of TTH and MTH.

\section{MATERIALS AND METHODS \\ Patient Cohorts}

All patients evaluated for TTH or MTH from May 2014 to July 2016 in the pediatric neurology clinics at the Lucile Packard Children's Hospital at Stanford University were retrospectively reviewed after approval by the institutional review board. Because this was a retrospective review, our institutional review board waived the requirement of informed consent. The study cohort was identified using the following inclusion criteria: age 18 years or younger and diagnostic criteria that fit either TTH or MTH as defined by International Classification of Headache Disorders, Version 3.0 (ICHD-3), excluding migraine with brain stem aura, hemiplegic migraine, and retinal migraine (Appendix). ${ }^{1}$ Only patients without any prior treatment with prescription medication who underwent diffusion-weighted MR imaging at 3T (as a standard protocol) following initial consultation but before the start of treatment were included. Prior neuroimaging findings must have been read as normal and included no incidental findings or abnormalities (eg, unidentified bright objects, developmental venous anomaly, or Chiari I abnormality). The patients had varying times between diagnosis and neuroimaging. Some patients had multiple neuroimaging examinations, in which case the most recent scan was selected for analysis to ensure consistency. Time to last headache was not collected (not feasible for this retrospective review), but patients' MR imaging intake records were reviewed to exclude patients with active headache or migraine.

Strict exclusion criteria were applied and comprised the following: inadequate data or image-registration quality, any concern for secondary headache, current or prior history of developmental delay, history of medication-overuse headache, underlying cardiac disease, epilepsy, prior or current hemorrhage, vascular lesions (aneurysm, AVM, fistula, or steno-occlusive disease), or prior strokes, given their potential impact on regional diffusion properties in the brain. Additionally, any patient with a previously diagnosed genetic, metabolic, or medical disease was excluded from this study. Patients with any focal neurologic findings, even if incidental, were excluded.

The control group consisted of 100 children with no known neurologic, neurocognitive, developmental, or behavioral deficits who had normal-appearing brains on MR imaging. Control subjects were imaged as a standard of care for evaluation of syncope, nausea, family history of aneurysm or cancers, scalp nevus, isolated facial lesions, orbital strabismus, cholesteatoma of the ear, any neurologic symptoms or deficits, sinus disease or inflammatory nasal obstruction, and familial short stature. This control group study population has been previously described. ${ }^{11}$

\section{MR Imaging Acquisition}

All subjects underwent brain MR imaging at 3T (Discovery 750W; GE Healthcare, Milwaukee, Wisconsin) with an 8-channel head coil on a single MR imaging scanner. Echo-planar whole-brain
DWI was acquired in all cases with $\mathrm{TR}=1500 \mathrm{~ms}, \mathrm{TE}=37 \mathrm{~ms}$, flip angle $=90^{\circ}, \mathrm{FOV}=24 \mathrm{~cm}^{2}$, acceleration factor $=2$, in-plane resolution $=0.94 \mathrm{~mm}^{2}$, acquisition matrix $=128 \times 128$ interpolated to a $256 \times 256$ matrix, 44 sections with $4-\mathrm{mm}$ slice thickness, no skip, 2 diffusion-weightings of $b=0 \mathrm{~s} / \mathrm{mm}^{2}$ and $b=1000$ $\mathrm{s} / \mathrm{mm}^{2}$, with diffusion gradients acquired in 3 directions averaged for the latter. ADC, derived from DWI, has demonstrated high reproducibility and was performed as part of routine institutional neuroimaging. ${ }^{12}$

\section{Image Processing}

A custom image-processing pipeline was used in this work to extract quantitative values of regional brain volume and ADC values, previously described in more detail by Forkert et al. ${ }^{11}$ Briefly described, after motion correction of the DWI dataset acquired with and without diffusion-weighting by rigid registration, the quantitative apparent diffusion coefficient parameter map was calculated using a Stejskal-Tanner equation. ${ }^{13}$ For regional diffusion and volumetric analysis, the Montreal Neurological Institute-152 brain atlas was nonlinearly registered to the DWI dataset and the resulting transformation was used to warp the Harvard-Oxford subcortical atlas brain regions to the subject-specific brain anatomy. ${ }^{14}$ Brain regions included in this brain atlas are the cerebral cortex, cerebral white matter, thalamus, caudate, putamen, globus pallidus, amygdala, hippocampus, brain stem, and nucleus accumbens. Two experienced observers checked all registration results to ensure suitable data and registration quality. The aligned brain atlas regions were then used to measure the corresponding regional brain volumes and median ADC values combined for corresponding brain structures in the left and right hemispheres, whereas the lateral ventricles were only used for volumetric assessment.

\section{Statistical Analysis}

Multivariate analysis of covariance was used for group comparison of the control group and children with MTH and TTH using the volumetric and median ADC values as dependent variables, age as a covariate, and the class (control/TTH/MTH) as the fixed factor. SPSS (Version 24.0, IBM, Armonk, NY) was used for MANCOVA statistical analyses. A $P$ value $<.05$ (Bonferronicorrected) was considered significant.

In addition to the statistical analysis described above, plots with age-related fifth, 10th, 25th, 50th, 75th, 90th, and 95th quantile curves determined using local piecewise regression analysis based on the quantitative analysis of the control cohort were generated individually for each brain structure and parameter along with the corresponding data points extracted from the children with MTH and TTH. ${ }^{15}$ The plots were generated using the R statistical software package of The R Foundation of Statistical Computing (Version 3.2.2; www.r-project.org).

\section{RESULTS}

\section{Clinical Characteristics}

A total of 214 patients with TTH or MTH were reviewed for inclusion in this study. Seventy-four patients (35\%) were excluded due to medical comorbidity; 51 (24\%), due to secondary or medication overuse-type headache; 25 (12\%), due to abnormal neu- 


\begin{tabular}{|c|c|c|c|}
\hline & $\begin{array}{l}\text { Control } \\
(n=100)\end{array}$ & $\begin{array}{l}\text { TTH } \\
(n=31)\end{array}$ & $\begin{array}{c}\text { MTH } \\
(n=23)\end{array}$ \\
\hline \multicolumn{4}{|l|}{ Demographics } \\
\hline Median age (range) (mo) & $98(4-213)$ & $99(24-209)$ & $83(12-212)$ \\
\hline $\operatorname{Sex}(M / F)$ & $36: 64$ & 13:18 & $6: 17$ \\
\hline Family history of any headache (No.) (\%) & & $6(19 \%)$ & $10(43 \%)$ \\
\hline \multicolumn{4}{|l|}{$\begin{array}{l}\left.\text { ADC on neuroimaging ([mean } \times 10^{-6} \mathrm{~mm}^{2} / \mathrm{s}\right] \text {, } \\
\text { standard error, }{ }^{\text {a }} P \text { value vs control) }\end{array}$} \\
\hline Cerebral cortex & $899.05(3.44)$ & $901.78(7.04)>.5$ & $907.29(8.22)>.5$ \\
\hline Thalamus & $812.48(3.51)$ & $815.44(6.26)>.5$ & $833.11(7.11) .045^{\mathrm{b}}$ \\
\hline Caudate & $815.16(3.98)$ & $821.42(7.11)>.5$ & $833.99(8.64) .149$ \\
\hline Putamen & $799.94(3.48)$ & $792.42(6.21)>.5$ & $800.75(7.55)>.5$ \\
\hline Pallidum & $836.62(4.05)$ & 822.09 (7.22) .24 & $838.30(8.78)>.5$ \\
\hline Hippocampus & $935.52(4.50)$ & $965.45(8.03) .013^{b}$ & $970.46(9.76) .038^{b}$ \\
\hline Amygdala & $885.83(3.79)$ & $882.75(6.78)>.5$ & $904.48(8.24) .113$ \\
\hline Nucleus accumbens & $856.83(4.72)$ & $841.45(8.43) .34$ & $850.75(8.43)>.5$ \\
\hline Brain stem & $806.72(3.43)$ & $825.01(6.13) .05^{\mathrm{b}}$ & $840.71(7.45)<.001^{\mathrm{b}}$ \\
\hline \multicolumn{4}{|l|}{$\begin{array}{l}\text { Volume on neuroimaging (mean [in } \mathrm{mL}] \\
\text { standard error, }{ }^{\text {a }} P \text { value vs control) }\end{array}$} \\
\hline Cerebral cortex & $344.33(4.19)$ & $350.56(3.92)>.5$ & 362.54 (9.08) .212 \\
\hline Thalamus & $6.62(0.08)$ & $5.54(0.12) .148$ & $5.71(0.15)>.5$ \\
\hline Caudate & $2.68(0.04)$ & $2.64(0.06)>.5$ & $2.71(0.08)>.5$ \\
\hline Putamen & $4.49(0.06)$ & $4.46(0.06)>.5$ & $4.63(0.12)>.5$ \\
\hline Pallidum & $1.46(0.02)$ & $1.42(0.03)>.5$ & $1.50(0.04)>.5$ \\
\hline Hippocampus & $3.10(0.04)$ & $3.07(0.07)>.5$ & $3.21(0.08)>.5$ \\
\hline Amygdala & $1.53(0.02)$ & $1.55(0.04)>.5$ & $1.59(0.04)>.5$ \\
\hline Nucleus accumbens & $0.37(0.01)$ & $0.38(0.04)>.5$ & $0.039(0.01)>.5$ \\
\hline Brain stem & $22.79(0.30)$ & $22.51(0.53) .108$ & $22.97(0.64)>.5$ \\
\hline
\end{tabular}

${ }^{a}$ Means and standard error were corrected to an age of 97 months in all groups.

b Significant.

roimaging findings; and 10 (5\%), due to developmental delay. Twenty-three patients with MTH (median age, 83 months; ranging from 30 to 212 months), 31 patients with TTH (median age, 99 months; ranging from 32 to 209 months), and 100 controls (median age, 98 months; ranging from 4 to 213 months) were included in this study. Thirteen males and 18 females were in the TTH group, and 6 males and 17 females were in the MTH group (Table). None of the patients had aura-preceding onset of symptoms at the time of diagnosis.

\section{Statistical Analysis}

The MANCOVA analysis using the volume and median ADC values as dependent variables, age as a covariate, and the class as a fixed factor revealed a statistically significant difference among patients with MTH, patients with TTH, and healthy controls $(P<$ .001). A secondary MANCOVA did not reveal a statistical difference between patients with MTH and patients with TTH, indicating that both headache types are represented by similar imaging findings compared with healthy controls regarding both volume and diffusion metrics.

In pair-wise analysis, no statistically significant differences regarding the volume of the cerebral cortex, cerebral white matter, thalamus, caudate, putamen, globus pallidus, amygdala, hippocampus, brain stem, and nucleus accumbens were found among patients with MTH, those with TTH, and controls $(P>$ $.135)$.

Post hoc pair-wise analysis of the median ADC values revealed significant differences in ADC for the hippocampus and brain stem. More precisely, patients with TTH $(P=.013)$ and those with $\mathrm{MTH}(P=.038)$ showed significantly increased ADC values in the hippocampus compared with healthy controls (age-corrected means: MTH, $962.6 \pm 9.6 \times 10^{-6} \mathrm{~mm}^{2} / \mathrm{s}$; TTH, $963.2 \pm$ $8.2 \times 10^{-6} \mathrm{~mm}^{2} / \mathrm{s}$; controls, $\left.935.8 \pm 4.6 \times 10^{-6} \mathrm{~mm}^{2} / \mathrm{s}\right)$. Likewise, patients with TTH $(P=.05)$ and patients with MTH $(P<$ $.001)$ showed significantly increased ADC values in the brain stem compared with healthy controls (age-corrected means: MTH, $838.3 \pm 7.2 \times 10^{-6} \mathrm{~mm}^{2} / \mathrm{s}$; TTH, 824.2.2 $\pm 6.1 \times 10^{-6} \mathrm{~mm}^{2} / \mathrm{s}$; controls, $807.2 \pm 3.4 \times 10^{-6} \mathrm{~mm}^{2} / \mathrm{s}$ ). Additionally, patients with MTH were noted to have increased ADC in the thalamus compared with controls $\left(P=.045 ; \mathrm{MTH}, 833.1 \pm 7.1 \times 10^{-6}\right.$ $\mathrm{mm}^{2} / \mathrm{s}$; controls, $\left.812.5 \pm 3.5 \times 10^{-6} \mathrm{~mm}^{2} / \mathrm{s}\right)$. No other parameter reached a statistically significant difference among groups (Table).

\section{Qualitative Analysis}

The results of the statistical evaluation can be also confirmed by visual analysis of the plots generated using local piecewise regression analysis (Figure). Overall, no obvious difference between patients with MTH (red dots) and TTH (blue boxes) is obvious for any parameter analyzed. The plots clearly show the significantly increased ADC values in the hippocampus and brain stem in patients with MTH and TTH compared with healthy controls, while no clear difference is obvious for any other parameter.

\section{DISCUSSION}

We hypothesized that patients with MTH may exhibit different diffusion values in the limbic and brain stem circuitry compared with those with TTH and healthy controls, given the underlying differences in pathophysiology of patients with MTH and TTH. Most interesting, this study identified increased diffusion patterns 

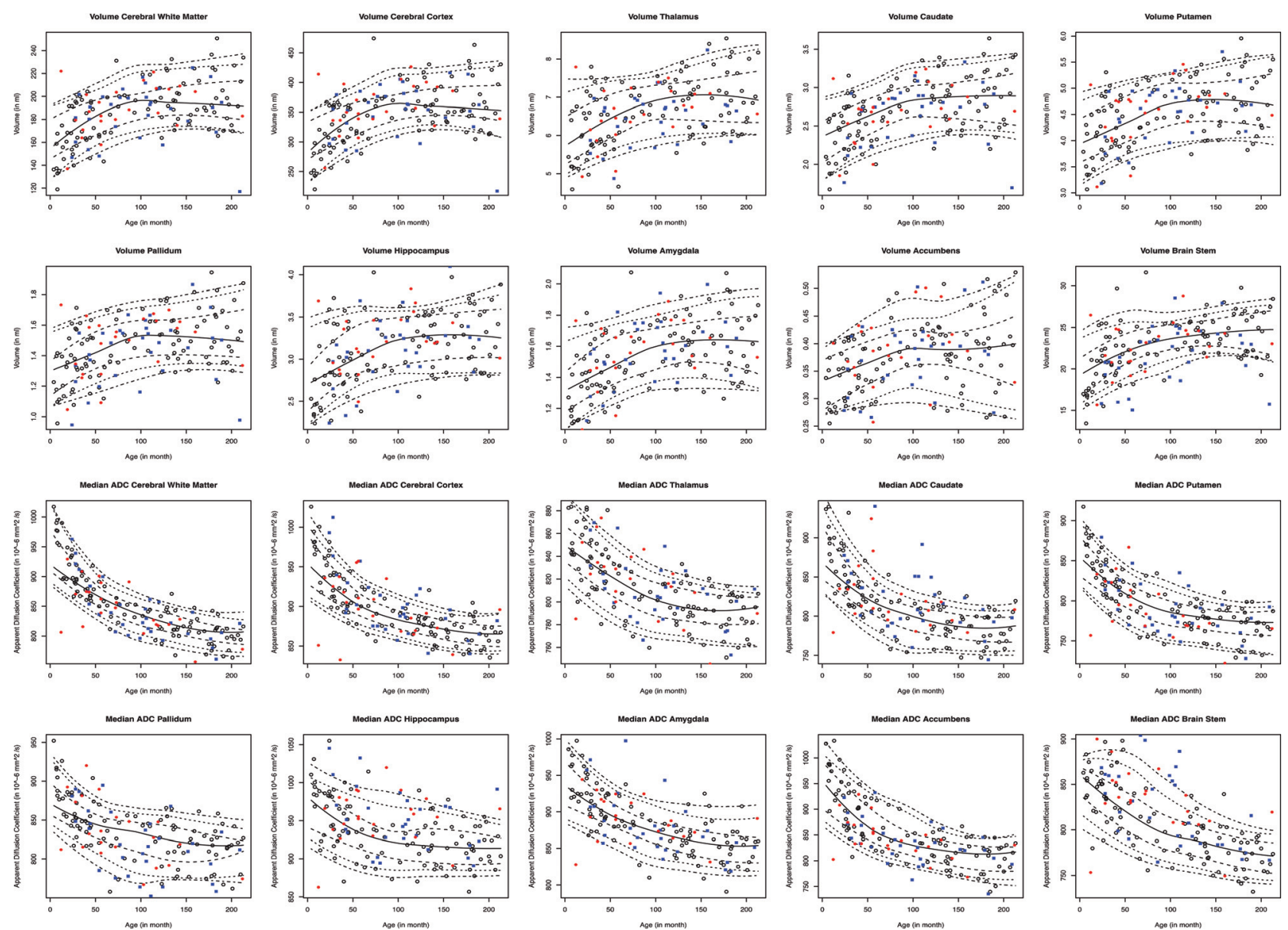

FIGURE. Visual analysis of all plots generated using local piecewise regression analysis for healthy control children (black circles) and patients with TTH (blue boxes) and MTH (red dots).

in those with both TTH and MTH in the hippocampus and brain stem as well as in the thalamus in only those with MTH compared with controls, without any concurrent volume abnormalities. These regions are involved with both pain nociception circuitry and the limbic system, which has been previously implicated in the perception of pain in adult patients with MTH at the functional, mechanistic, and electrophysiologic levels. ${ }^{16,17}$ The involvement of TTH abnormalities, which was unexpected, indicates that diffusion abnormalities in the hippocampus and thalamus may potentially reflect enhanced pain circuitry as opposed to intrinsic pathophysiologic differences between MTH and TTH.

This study is the first to describe early ADC diffusion changes in several areas involved in the limbic circuit in pediatric patients with MTH and TTH. While no volumetric abnormalities were identified, the median age of patients with MTH and TTH was 83 months and 99 months, respectively, which is younger than the median age of 168 months in a prior study. ${ }^{8}$ This finding may explain the lack of volumetric change in our cohort. ${ }^{8}$ Therefore, the difference in age between these 2 study groups may suggest that the early diffusion changes found in this work may become more pronounced with continued headaches and manifest as volumetric abnormalities into adolescence and adulthood. ${ }^{5-8}$

Of specific interest in our study was the involvement of the brain stem in patients with MTH and TTH. Mechanistically, this area of the brain has bidirectional connections between the diencephalic structures and the trigeminovascular system, which could either be activated by these areas or be a primary pacer for activation of these structures. ${ }^{18}$ Increased flow and activation of the brain stem on PET imaging have been previously observed in adult patients with $\mathrm{MTH}$, indicating another potential component in MTH pain circuitry. ${ }^{19}$ Finally, while this area has been identified in previous studies to be functionally activated in acute migraine, long-standing diffusion changes have not been previously identified in pediatric patients. ${ }^{19,20}$

The hippocampus has been previously noted to exhibit reduced volume in adult patients with high-frequency TTH and MTH. ${ }^{21,22}$ The thalamus seemed to be only significantly altered in patients with MTH in this study, though recently published reports have identified the significance of this brain region as a functional pain-signaling relay. ${ }^{23,24}$ Additionally, the thalamus volume has been found to be altered in patients with medication overuse headache and MTH with and without aura. ${ }^{23-25}$ The amygdala has also been demonstrated to have volumetric decreases in patients with chronic MTH, demonstrating an inverse relationship to the frequency of migraine. ${ }^{26}$ These structures are more prominently implicated in the limbic circuitry, which is presumed to be more related to pain perception than pain sensation. ${ }^{18,24}$ Both the hippocampus and amygdala are bidirectionally linked to the brain stem and thalamus; thus, the involvement of 
these structures in our study may demonstrate functional preanatomic linkage in migraine physiology. Our findings may indicate a propensity toward volumetric abnormalities in adulthood in patients previously reported with long-standing diffusion abnormalities beginning in childhood, though this propensity requires further investigation. The isolated significance of the thalamus in MTH compared with TTH and controls in our study is consistent with prior studies that have not demonstrated involvement of this region in TTH and may be a more specific factor in migraine, given previously established thalamocortical circuitry in experience processing. ${ }^{25,27}$

There are several limitations to this study. As a retrospective case-control study, we can only speculate on differences at a single time point. Both the TTH and MTH groups are small, given that this is a single-center study, but the large number of healthy controls allows a unique comparison. Additionally, although validated in pediatric patients, the International Classification of Headache Disorders, Version 3.0 criteria has come under scrutiny for both under- and overdiagnosis of paroxysmal pain disorders in this patient population. ${ }^{28}$ As a retrospective review, there is the risk of diagnostic inaccuracy, specifically less severe migraine headache diagnosed as tension-type headache. Furthermore, there are no clinical data to correlate the imaging findings with headache severity, because more severe or protracted headaches could potentially demonstrate greater diffusion changes. Furthermore, our inclusion criteria of head imaging before starting treatment might result in a bias toward more severe headache phenotypes. Because previous studies have indicated that diffusion findings may be transient in adults, whether the patient was having a headache at the time of scanning or near the time of scanning may affect the quantitative imaging findings. ${ }^{5,9,10}$ For analysis, we combined data from both the left and right hemispheres to reduce the number of hypotheses tested, which omitted trends in laterality. In addition, we recognize that segmentation of small cerebral structures can be imperfect, but an automated approach (with visual quality control) was used to ensure reproducibility, given that manual segmentation is prone to observer bias. A final limitation of this study is that we did not assess patients with MTH with aura. This assessment was not performed due to the lower prevalence of "pure" MTH with aura and higher rates of mixedtype MTH in pediatric populations. ${ }^{29-31}$

\section{CONCLUSIONS}

This study identifies early cerebral diffusion changes in patients with TTH and MTH compared with healthy children. The hypothesized underlying pathophysiologic mechanisms of nociception in MTH and TTH may help explain this finding and may be a precursor to the structural changes seen in adult patients with chronic pain syndromes and MTH.

\section{APPENDIX}

\section{Criteria for Diagnosis of Tension-Type Headache}

1) Infrequent episodic tension-type headache

a) At least 10 episodes of headache occurring on $<1$ day per month on average ( $<12$ days per year) and fulfilling criteria $b-d$

b) Lasting from 30 minutes to 7 days

c) At least 2 of the following 4 characteristics: i) Bilateral location

ii) Pressing or tightening (nonpulsating) quality

iii) Mild or moderate intensity

iv) Not aggravated by routine physical activity such as walking or climbing stairs

d) Both of the following criteria:

i) No nausea or vomiting

ii) No more than 1 of photophobia or phonophobia

e) Not better accounted for by another ICHD-3 diagnosis

2) Frequent episodic tension-type headache

a) At least 10 episodes of headache occurring on 1-14 days per month on average for $>3$ months ( $>12$ and $<180$ days per year) and fulfilling criteria $\mathrm{b}-\mathrm{d}$

b) Lasting 30 minutes to 7 days

c) At least 2 of the following 4 characteristics:

i) Bilateral location

ii) Pressing or tightening (nonpulsating) quality

iii) Mild or moderate intensity

iv) Not aggravated by routine physical activity such as walking or climbing stairs

d) Both of the following criteria:

i) No nausea or vomiting

ii) No more than 1 of photophobia or phonophobia

e) Not better accounted for by another ICHD-3 diagnosis

3) Chronic tension-type headache

a) Headache occurring on $>15$ days per month on average for $>3$ months ( $>180$ days per year) and fulfilling criteria $b-d$

b) Lasting hours to days, or unremitting

c) At least 2 of the following 4 characteristics:

i) Bilateral location

ii) Pressing or tightening (nonpulsating) quality

iii) Mild or moderate intensity

iv) Not aggravated by routine physical activity such as walking or climbing stairs

d) Both of the following criteria:

i) No nausea or vomiting

ii) No more than 1 of photophobia or phonophobia

e) Not better accounted for by another ICHD-3 diagnosis

4) Probable tension-type headache

a) Tension-type-like headache missing 1 of the features required to fulfill all criteria for a subtype of tension-type headache coded above, and not fulfilling the criteria for another headache disorder.

\section{Criteria for Diagnosis of Migraine-Type Headache}

1) Migraine without aura

a) At least 5 attacks fulfilling criteria b-d

b) Headache attacks lasting 4-72 hours (untreated or unsuccessfully treated)

c) Headache has at least 2 of the following 4 characteristics:

i) Unilateral location

ii) Pulsating quality

iii) Moderate or severe pain intensity

iv) Aggravation by or causing avoidance of routine physical activity (eg, walking or climbing stairs)

d) During headache at least 1 of the following:

i) Nausea and/or vomiting

AJNR Am J Neuroradiol 39:935-41 May 2018 www.ajnr.org 
ii) Photophobia and phonophobia

e) Not better accounted for by another ICHD-3 diagnosis

2) Migraine with aura

a) At least 2 attacks fulfilling criteria b and c

b) One or more of the following fully reversible aura symptoms:

i) Visual

ii) Sensory

iii) Speech and/or language

iv) Motor

v) Brain stem

vi) Retinal

c) At least 2 of the following 4 characteristics:

i) At least 1 aura symptom spreading gradually for $>5 \mathrm{~min}$ utes and/or $\geq 2$ symptoms occurring in succession

ii) Each individual aura symptom lasting 5-60 minutes

iii) At least 1 unilateral aura symptom

iv) The aura accompanied or followed within 60 minutes by headache

d) Not better accounted for by another ICHD-3 diagnosis, and transient ischemic attack excluded

3) Chronic migraine

a) Headache (tension-type-like and/or migraine-like) on $>15$ days per month for $>3$ months and fulfilling criteria $b$ and $c$

b) Occurring in a patient who has had at least 5 attacks fulfilling criteria $b-d$ for either migraine without aura and/or criteria $b$ and $\mathrm{c}$ for migraine with aura

c) On $>8$ days per month for $>3$ months, fulfilling any of the following:

i) Criteria $\mathrm{c}$ and d for migraine without aura

ii) Criteria $\mathrm{b}$ and $\mathrm{c}$ for migraine with aura

iii) Believed by the patient to be migraine at onset and relieved by a triptan or ergot derivative

d) Not better accounted for by another ICHD-3 diagnosis

4) Probable migraine

a) Attacks fulfilling all but 1 of the criteria a-d for migraine without aura or all but 1 of the criteria $\mathrm{a}-\mathrm{c}$ for migraine with aura

b) Not fulfilling the ICHD-3 criteria for any other headache disorder

c) Not better accounted for by another ICHD-3 diagnosis.

\section{ACKNOWLEDGMENTS}

We wish to thank the Division of Child Neurology for their support in the production of this article.

Disclosures: Nils Daniel Forkert-UNRELATED: Grants/Grants Pending: Natural Sciences and Engineering Research Council of Canada; National Institutes of Health, Heart and Stroke Foundation; MS Society. * *Money paid to the institution.

\section{REFERENCES}

1. Headache Classification Committee of the International Headache Society (IHS). International Classification of Headache Disorders, 3rd edition (beta version). Cephalalgia 2013:33:629-808 CrossRef Medline

2. Lewis DW, Dorbad D. The utility of neuroimaging in the evaluation of children with migraine or chronic daily headache who have normal neurologic examinations. Headache 2000;40:629-32 CrossRef Medline

3. Schwedt TJ, Guo Y, Rothner AD. “Benign” imaging abnormalities in children and adolescents with headache. Headache 2006;46:387-98 CrossRef Medline

4. Fazekas F, Koch M, Schmidt R, et al. The prevalence of cerebral damage varies with migraine type: a MRI study. Headache 1992;32: 287-91 Medline

5. Jäger HR, Griffin NJ, Goadsby PJ. Diffusion and perfusion weighted MR imaging in persistent migrainous visual disturbances. Cephalagia 2005;25:323-32 Medline

6. Oberndorfer S, Wober C, Nasel C, et al. Familial hemiplegic migraine: follow-up findings of diffusion-weighted magnetic resonance imaging (MRI), perfusion-MRI and [99mTc] HMPAOSPECT in a patient with prolonged hemiplegic aura. Cephalalgia 2004;24:533-39 Medline

7. Rocca MA, Colombo B, Pratesi A, et al. A magnetization transfer imaging study of the brain in patients with migraine. Neurology 2000;54:507-09 Medline

8. Messina R, Rocca MA, Colombo B, et al. White matter microstructure abnormalities in pediatric migraine patients. Cephalalgia 2015; 35:1278-86 CrossRef Medline

9. Degirmenci B, Yaman M, Haktanir A, et al. Cerebral and cerebellar ADC values during a migraine attack. Neuroradiology 2007;49: 419-26 CrossRef Medline

10. Belvís R, Ramos R, Villa $C$, et al. Brain apparent water diffusion coefficient magnetic resonance imaging during a prolonged visual aura. Headache 2010;50:1045-49 CrossRef Medline

11. Forkert ND, Li MD, Lober RM, et al. Gray matter growth is accompanied by increasing blood flow and decreasing apparent diffusion coefficient during childhood. AJNR Am J Neuroradiol 2016;37: 1738-44 CrossRef Medline

12. Grech-Sollars M, Hales PW, Miyazaki K, et al. Multi-centre reproducibility of diffusion MRI parameters for clinical sequences in the brain. NMR Biomed 2015;28:468-85 CrossRef Medline

13. Stejskal EO, Tanner JE. Spin diffusion measurements: spin echoes in the presence of a time-dependent field gradient. Journal of Chemical Physics 1965;42:288-92

14. Mazziotta J, Toga A, Evans A, et al. A probabilistic atlas and reference system for the human brain: International Consortium for Brain Mapping (ICBM). Philos Trans R Soc Lond B Biol Sci 2001;356: 1293-322 CrossRef Medline

15. Sakov A, Golani I, Lipkind D, et al. High-throughput data analysis in behavior genetics. Ann Appl Stat 2010;4:743-63 CrossRef

16. Wilcox SL, Veggeberg R, Lemme J, et al. Increased functional activation of limbic brain regions during negative emotional processing in migraine. Front Hum Neurosci 2016;10:366 CrossRef Medline

17. Demarquay G, Mauguière F. Central nervous system underpinnings of sensory hypersensitivity in migraine: insights from neuroimaging and electrophysiological studies. Headache 2016;56:1418-38 CrossRef Medline

18. Akerman S, Holland PR, Goadsby PJ. Diencephalic and brainstem mechanisms in migraine. Nat Rev Neurosci 2011;12:570-84 CrossRef Medline

19. Cutrer FM, O’Donnell A, Sanchez del Rio M. Functional neuroimaging: enhanced understanding of migraine pathophysiology. Neurology 2000;55(9 suppl 2):S36-45 Medline

20. Weiller C, May A, Limmroth V, et al. Brain stem activation in spontaneous human migraine attacks. Nat Med 1995;1:658-60 CrossRef Medline

21. Maleki N, Becerra L, Brawn J, et al. Common hippocampal structural and functional changes in migraine. Brain Struct Funct 2013; 218:903-12 CrossRef Medline

22. Schmidt-Wilcke T, Leinisch E, Straube A, et al. Gray matter decrease in patients with chronic tension type headache. Neurology 2005;65: 1483-86 CrossRef Medline

23. Amin FM, Hougaard A, Magon S, et al. Altered thalamic connectivity during spontaneous attacks of migraine without aura: a resting state fMRI study. Cephalalgia 2017 Jan 1. [Epub ahead of print] CrossRef Medline

24. Magon S, May A, Stankewitz A, et al. Morphological abnormalities 
of thalamic subnuclei in migraine: a multicenter MRI study at 3 Tesla. J Neurosci 2015;35:13800-06 CrossRef Medline

25. Chen Z, Jia Z, Chen X, et al. Volumetric abnormalities of thalamic subnuclei in medication overuse headache. J Headache Pain 2017; 18:82 CrossRef Medline

26. Schmitz N, Admiraal-Behloul F, Arkink EB, et al. Attack frequency and disease duration as indicators for brain damage in migraine. Headache 2008;48:1044-55 CrossRef Medline

27. Coppola G, Di Renzo A, Tinelli E, et al. Thalamo-cortical network activity between migraine attacks: insights from MRI-based microstructural and functional resting-state network correlation analysis. J Headache Pain 2016;17:100 CrossRef Medline

28. Genizi J, Khourieh Matar A, Zelnik N, et al. Frequency of pediatric migraine with aura in a clinic-based sample. Headache 2016;56: 113-17 CrossRef Medline

29. Split W, Neuman W. Epidemiology of migraine among students from randomly selected secondary schools in Lodz. Headache 1999; 39:494-501 CrossRef Medline

30. Zwart JA, Dyb G, Holmen TL, et al. The prevalence of migraine and tension-type headaches among adolescents in Norway: the NordTrøndelag Health Study (Head-HUNT-Youth), a large populationbased epidemiological study. Cephalalgia 2004;24:373-79 CrossRef Medline

31. Fendrich K, Vennemann M, Pfaffenrath V, et al. Headache prevalence among adolescents: the German DMKG headache study. Cephalalgia 2007;27:347-54 CrossRef Medline 